\title{
Предикторы успешности краудфандингового проекта
}

М.Н. ГОРДЕЕВ, Национальный исследовательский университет «Высшая школа экономики», Москва. E-mail: mngordeev@gmail.com

Аннотация. В работе методом корреляционно-регрессионного анализа исследованы факторы, которые оказывают влияние на успешный сбор средств через механизм краудфандинга. Изучение данных о более чем 12 тыс. проектов, проведенных в России, позволило выявить связь успешности проекта с параметрами, задаваемыми автором при его запуске, а также информационным сопровождением по мере его реализации. В ходе исследования обнаружен эффект обучения, который проявляется во влиянии опыта автора проекта в краудфандинге на реализацию последующих инициатив, установлена корреляция между успешностью коммерческих инициатив и соответствием вознаграждения взносу спонсора. Полученные данные позволяют глубже понять механизм краудфандинга и лежащие в его основе процессы. Особое внимание уделено использованию краудфандинга в качестве инструмента финансирования молодых компаний и стартапов.

Ключевые слова: краудфандинг; народное финансирование; успешность проекта; Planeta.ru

Проведенное в 2018 г. исследование отечественных стартапов подтвердило, что главной проблемой, с которой сталкиваются молодые инновационные компании, является недостаток финансирования [Соловьев, 2018. С.14-15]. Действительно, сложившаяся политическая и экономическая ситуация подталкивает инвесторов и кредиторов к выбору наиболее надежных объектов для вложения средств, так что привлечение финансирования в высокорисковые инновационные компании, которые только начинают свое функционирование, становится трудновыполнимой задачей. Таким образом, приобретает актуальность использование нетрадиционных источников финансирования, одним из которых является краудфандинг.

Механизм краудфандинга состоит в том, что автор инициативы размещает на специальной интернет-площадке (крауд-платформе) информацию о запланированном проекте с целью сбора средств, необходимых для реализации проекта (целевой суммы). Заинтересованные спонсоры перечисляют свои средства на счет крауд-платформы в обмен на обязательство автора направить им заранее оговоренное вознаграждение. Как правило, накопленная сумма передается автору на реализацию проекта только тогда, 
когда она достигает целевого значения, в противном случае средства возвращаются спонсорам.

Этот механизм обладает рядом преимуществ. Так, вместе со сбором средств происходит апробация востребованности идеи аудиторией и автоматически отсеиваются бесперспективные начинания. В ряде работ показано, что такого рода фильтр подчас оказывается более эффективным, чем отбор проектов профессиональными инвесторами [Mollick et al., 2016a. C.12]. При этом из процесса инвестирования исключается промежуточное звено (банки или венчурные фонды), так что собственники средств могут самостоятельно определять, во что их вкладывать [Lynn, 2013. С.2; Амосов, 2014]. Кроме того, зарубежными исследователями показано, что краудфандинг является эффективным источником финансирования стартапов, внедряющих инновации [Chan, 2017; Schwienbacher, Larralde, 2012], в частности, в результате реализации таких проектов было зарегистрировано несколько тысяч патентов [Mollick et al., 2016b].

Залогом успешности реализации большинства венчурных проектов являются три составляющие: 1) качество и востребованность самой идеи 2) способность автора ее реализовать и 3) способность донести информацию об этом до инвесторов, чтобы привлечь средства для воплощения идеи в жизнь. Первые два компонента определяются слишком большим многообразием предпринимательских инициатив, тогда как третий обнаруживает определенные закономерности и подробно изучен для традиционных инструментов финансирования [Shane, Cable, 2002; Hsu, 2007]. Однако в случае краудфандинга мы имеем дело с весьма специфическим инвестором - широким кругом рядовых граждан, которые не специализируются на инвестициях, и, как правило, руководствуются иными мотивами, помимо извлечения прибыли. Таким образом, изучение механизмов взаимодействия с этой аудиторией становится самостоятельной научной проблемой. В зарубежной литературе предпринимались попытки изучения этих процессов и, в частности, было определено, что вероятность успешного сбора средств на проект во многом зависит от параметров, задаваемых при его запуске, а также от последующего информационного сопровождения [Mollick, 2014. C.8; Strickler, 2011; Cordova at el., 2015. C.119]. 
Цель настоящей работы - углубление знаний в этой области, а также их перенесение на случай российской аудитории. Объектом исследования выступают российские краудфандинговые проекты, т.е. проекты по сбору средств на реализацию венчурных инициатив. Предметом исследования являются предикторы успешности краудфандинговых проектов. Термин «успешность» в рамках настоящей статьи относится только к процессу сбора средств и не охватывает последующее непосредственное воплощение идеи в жизнь.

Эмпирической основой послужили общедоступные данные о проектах, размещенных на одной из крупнейших российских крауд-платформ Planeta.ru (URL: https://Planeta.ru.ru/), выгруженные программными средствами в формате JSON 12 декабря 2018 г. По всем проектам рассматривались 14 унифицированных параметров, среди которых длительность, даты проведения, целевая и собранная сумма, число спонсоров, имя автора и число проведенных им проектов и т.д. Полнота выборки контролировалась по размеру общей собранной суммы на проекты платформы: по данным Planeta.ru, на указанную дату она составила 953,7 млн руб., общий объем финансирования, привлеченный под проекты из нашей выборки, - 951,6 млн руб. (расхождение 0,2\%). Причины расхождения: продолжительный период выгрузки данных (около шести часов), недоступность информации по некоторым проектам, скрытым от общего доступа. Было проанализировано 12278 проектов, по которым на указанную дату был завершен сбор средств. Их усредненные и медианные (в скобках) характеристики представлены в таблице 1.

В отличие от общепринятой практики, крауд-платформа Planeta.ru позволяет получить средства авторам проектов, набравшим хотя бы $50 \%$ и более от размера целевой суммы - эти проекты и отнесены к успешным в нашем исследовании. При этом подразумевается, что автор проекта несет все взятые на себя обязательства по вознаграждению спонсоров.

Как видим по таблице 1, 28\% проектов (3407 ед.) закончились успешно (собрали 50\% и более от целевой суммы). Математическое среднее уровня финансирования оказалось вблизи $100 \%$, однако реальный разброс значений по этому параметру колеблется от 50\% до 2000\%. Следует также отметить значительный разброс размеров целевой суммы: от 5 тыс. до 15 млн руб., что в среднем дало примерно 240 тыс. руб. 
Таблица 1. Основные параметры проектов, проведенных на краудплатформе Planeta.ru c 14.05.2012 г. по 12.12.2018 г.

\begin{tabular}{|l|c|c|c|}
\hline \multicolumn{1}{|c|}{ Параметр } & Все проекты & $\begin{array}{c}\text { Успешные } \\
\text { проекты }\end{array}$ & $\begin{array}{c}\text { Безуспешные } \\
\text { проекты }\end{array}$ \\
\hline Цель проекта, руб. & $\begin{array}{c}358169 \\
(157925)\end{array}$ & $\begin{array}{c}240679 \\
(120000)\end{array}$ & $\begin{array}{c}403292 \\
(190000)\end{array}$ \\
\hline Собрано средств, руб. & 74380 & 241784 & 10087 \\
& $(4300)$ & $(110113)$ & $(1000)$ \\
\hline Уровень финансирования,\% & 31 & 103 & 4 \\
& $(3)$ & $(100)$ & $(1)$ \\
\hline Количество спонсоров & 41 & 140 & 4 \\
& $(0)$ & $(67)$ & $(0)$ \\
\hline Средний взнос отдельного & 865 & 2822 & 113 \\
спонсора, руб. & $(0)$ & $(1531)$ & 75 \\
\hline Длительность процесса сбо- & 76 & 79 & $(65)$ \\
ра денег, дней & $(65)$ & $(63)$ & 8871 \\
\hline Количество проектов & 12278 & 3407 & \\
\hline
\end{tabular}

Интересно, что «безуспешные» проекты смогли привлечь в среднем лишь 4\% от целевой суммы (по окончании периода сбора средств все они возвращаются спонсорам). При этом почти половина из них (49\%) не привлекли средств вовсе, а еще $37 \%$ не достигли даже $1 / 10$ от целевой суммы.

Как свидетельствует опыт зарубежных площадок, оптимальная длительность сбора средств составляет 30 дней, проекты со сроком 60 дней заметно реже становятся успешными [Strickler, 2011; Mollick, 2014. C.8], хотя некоторые исследователи [Cordova et al., 2015. C.119], напротив, заявляют о наличии положительной зависимости успешности проекта от длительности сбора средств. Очевидна необходимость дальнейшего изучения этого вопроса для прояснения характера влияния. У проектов, участвующих в нашем исследовании, средний срок составил около 2-2,5 месяцев, однако и в этом случае велик разброс значений: от 1 до 1416 дней.

В работах зарубежных авторов отмечается также отрицательное влияние на успешность сбора средств размера целевой суммы [Cordova et al., 2015. C.119], и положительное - наличия видео и количества оповещений автора о ходе проекта [Mollick, 2014. С.8].

На крауд-площадке Planeta.ru размещаются проекты по различным тематикам, которые разбиты на 16 категорий: среди них бизнес, наука и технологии, кино и видео, театр, музыка, 
благотворительность и др. Одним из обязательных условий размещения является общественная значимость проекта, что, впрочем, не исключает наличие у него коммерческой составляющей. В зависимости от преобладания той или иной компоненты проекты можно разделить на преимущественно коммерческие и преимущественно общественные. Несмотря на то, что такое разграничение довольно субъективно, существуют исследования [Салтыков, Гордеев, 2016. С. 54], показывающие, что проекты из этих двух категорий обнаруживают отличительные характеристики. Поэтому в настоящей работе анализ проводился по отдельности для каждой из подгрупп: к коммерческим мы отнесли 6610 проектов из нашей выборки, к общественным - 5668.

Одно из явных различий коммерческих и социальных проектов - характер вознаграждения. Поскольку основной целью общественных инициатив является создание некоего общего блага, в качестве вознаграждения в таких проектах чаще всего выступают благодарность авторов и некоторые символические подарки. Напротив, в коммерческих проектах создается коммерчески привлекательный продукт (работа/услуга), и чаще всего именно он и предлагается в качестве вознаграждения спонсорам. Вместе с тем на площадке Planeta.ru мы выявили ряд коммерческих инициатив, в которых предлагаемые вознаграждения были символическими или их стоимость значительно уступала размеру вклада спонсора (например, пластиковая авторучка за 800-1000 руб., керамическая кружка за 1800-2000 руб. и т.п.). Для того чтобы оценить степень влияния на успешность коммерческих проектов явного несоответствия вознаграждения спонсорскому вкладу, мы ввели и проанализировали переменную «Соответствие вознаграждения».

На основе анализа имеющихся теоретических исследований [Mollick, 2014. C.8; Strickler, 2011; Cordova et al., 2015. C.119] и накопленного практического опыта (табл. 1) были выдвинуты следующие гипотезы.

H1. Вероятность успешного сбора средств в краудфандинговом проекте зависит от его параметров, а именно:

a) она обратно пропорциональна размеру целевой суммы и прямо пропорциональна:

б) количеству поддержавших его спонсоров;

в) среднему размеру взноса отдельного спонсора;

г) длительности сбора средств на проект. 
H2. Вероятность успешного сбора средств в краудфандинговом проекте зависит от качества информационного сопровождения в период сбора средств, а именно:

a) она прямо пропорциональна количеству оповещений автора о ходе проекта и б) количеству комментариев по проекту;

в) она увеличивается при наличии заглавного видео.

H3. Вероятность успешного сбора средств в краудфандинговом проекте зависит от предшествующего опыта организации краудфандинговых проектов автором.

H4. Для коммерческих проектов соответствие вознаграждения размеру взноса спонсора положительно влияет на вероятность успешного сбора средств.

Для оценки качества информационного сопровождения проекта, отмеченного в гипотезе $\mathbf{H 2}$, использовались следующие характеристики: количество оповещений автора о ходе проекта, которое характеризует интенсивность актуализации данных и доведения их до аудитории; количество комментариев по проекту, которое характеризует активность обсуждения и качество выстраивания коммуникации с аудиторией; наличие заглавного видео в описании проекта, которое характеризует степень предварительной проработки проекта автором и облегчает получение информации потенциальными спонсорами.

\section{Результаты исследования}

Чтобы выяснить, в какой степени различные детерминанты оказывают влияние на успешность сбора средств в краудфандинговых проектах, мы провели корреляционно-регрессионный анализ данных. В таблице 2 приведены описательные статистики 12 изучаемых переменных: зависимой переменной выступало фактическое достижение порогового значения в 50\% от целевой суммы (бинарная переменная: 0-безуспешный, 1-успешный). В качестве независимых переменных рассматривались параметры проекта (целевая сумма, длительность, количество спонсоров, размер среднего взноса), качество информационного сопровождения (наличие видео (0-нет, 1-есть), количество новостей и комментариев), наличие у автора предшествующего опыта краудфандинга (успешного, негативного и опыта вообще (бинарные переменные: 0-нет, 1-есть)), а также соответствие вознаграждения взносу (0-не соответствует, 1-соответствует). 
Таблица 2. Описательные статистики зависимых и независимых переменных, использованных в анализе

\begin{tabular}{|c|l|c|c|c|c|}
\hline \multirow{2}{2}{} & \multicolumn{4}{|c|}{ Описательные статистики } \\
\cline { 3 - 6 } & Мин & Макс & Среднее & SD \\
\hline 1 & Успешность & 0 & 1 & 0,28 & 0,45 \\
\hline 2 & Целевая сумма, руб. & 5000 & 99999999 & 358169 & 1228222 \\
\hline 3 & Длительность сбора средств, дней & 1 & 1416 & 76 & 54,87 \\
\hline 4 & Количество спонсоров & 0 & 10994 & 41 & 186,93 \\
\hline 5 & Средний взнос отдельного спонсора, руб. & 0 & 150000 & 865 & 3454,25 \\
\hline 6 & Наличие в описании заглавного видео & 0 & 1 & 0,56 & 0,50 \\
\hline 7 & Количество новостей & 0 & 260 & 3,23 & 8,29 \\
\hline 8 & Количество комментариев & 0 & 1845 & 5,56 & 37,99 \\
\hline 9 & Наличие у автора опыта в краудфандинге & 0 & 1 & 0,17 & 0,38 \\
\hline 10 & $\begin{array}{l}\text { Наличие у автора хотя бы одного безуспеш- } \\
\text { ного проекта }\end{array}$ & 0 & 1 & 0,10 & 0,30 \\
\hline 11 & $\begin{array}{l}\text { Наличие у автора хотя бы одного успешного } \\
\text { проекта }\end{array}$ & 0 & 1 & 0,12 & 0,33 \\
\hline 12 & Соответствие вознаграждения & 0 & 1 & 0,96 & 0,20 \\
\hline
\end{tabular}

Примечание: SD - стандартное отклонение

Была рассчитана корреляция между всеми названными переменными (в таблице 3 приведена корреляционная матрица, порядковый номер переменных в которой соответствует нумерации таблицы 2).

\section{Таблица 3. Корреляционная матрица}

\begin{tabular}{|l|c|c|c|c|c|c|c|c|c|c|c|c|}
\hline & $\mathbf{1}$ & $\mathbf{2}$ & $\mathbf{3}$ & $\mathbf{4}$ & $\mathbf{5}$ & $\mathbf{6}$ & $\mathbf{7}$ & $\mathbf{8}$ & $\mathbf{9}$ & $\mathbf{1 0}$ & $\mathbf{1 1}$ & $\mathbf{1 2}$ \\
\hline 1 & 1 & & & & & & & & & & & \\
\hline 2 & $-0,06^{\star}$ & 1 & & & & & & & & & & \\
\hline 3 & $0,04^{\star}$ & $0,08^{\star}$ & 1 & & & & & & & & & \\
\hline 4 & $0,33^{\star}$ & $0,08^{\star}$ & $0,08^{\star}$ & 1 & & & & & & & & \\
\hline 5 & $0,35^{\star}$ & 0,01 & 0,00 & $0,05^{\star}$ & 1 & & & & & & & \\
\hline 6 & $0,09^{\star}$ & $0,04^{\star}$ & $0,05^{\star}$ & $0,07^{\star}$ & $0,03^{\star}$ & 1 & & & & & & \\
\hline 7 & $0,35^{\star}$ & 0,02 & $0,19^{\star}$ & $0,24^{\star}$ & $0,10^{\star}$ & $0,11^{\star}$ & 1 & & & & & \\
\hline 8 & $0,18^{\star}$ & $0,05^{\star}$ & $0,12^{\star}$ & $0,42^{\star}$ & 0,03 & $0,07^{\star}$ & $0,19^{\star}$ & 1 & & & & \\
\hline 9 & $0,26^{\star}$ & 0,00 & 0,02 & $0,13^{\star}$ & $0,11^{\star}$ & $-0,06^{\star}$ & $0,15^{\star}$ & $0,06^{\star}$ & 1 & & & \\
\hline 10 & $0,07^{\star}$ & 0,01 & $-0,01$ & 0,01 & $0,07^{\star}$ & $-0,10^{\star}$ & $0,05^{\star}$ & $-0,02$ & $0,73^{\star}$ & 1 & & \\
\hline 11 & $0,31^{\star}$ & $-0,01$ & $0,04^{\star}$ & $0,17^{\star}$ & $0,11^{\star}$ & $-0,04^{\star}$ & $0,17^{\star}$ & $0,08^{\star}$ & $0,81^{\star}$ & $0,37^{\star}$ & 1 & \\
\hline 12 & $0,12^{\star}$ & $-0,04^{\star}$ & 0,02 & $0,05^{\star}$ & $0,05^{\star}$ & $-0,00$ & $0,06^{\star}$ & 0,02 & $0,06^{\star}$ & $0,03^{\star}$ & $0,07^{\star}$ & 1 \\
\hline
\end{tabular}

Примечание: * $p<0.001$

Проведено тестирование на наличие мультиколлинеарности: значение фактора инфляции дисперсии (variance inflation factor, 
VIF) ни для одной из переменных не превысило 1,5, что свидетельствует об отсутствии мультиколлинеарности (пороговым значением, сигнализирующим о ее наличии, принято считать 10 [Field, 2005]) ${ }^{1}$.

\section{Результаты регрессионного анализа}

Всего было построено две регрессионные модели: первая описывает влияние параметров самого проекта, а вторая - факторов, связанных с информационным сопровождением проекта. Для больших числовых величин в этих моделях были использованы значения их натурального логарифма.

Тестируемое уравнение регрессии для обоих моделей:

$\mathrm{Y}=\beta_{0}+\beta_{1} \mathrm{X}_{1}+\beta_{2} \mathrm{X}_{2}+\beta_{3} \mathrm{X}_{3}+\beta_{4} \mathrm{X}_{4}+\varepsilon$,

где $\mathrm{Y}$ - зависимая переменная, успешность краудфандингового проекта;

$\beta_{0}-\beta_{4}-$ коэффициенты регрессии;

$\mathrm{X}_{1}-\mathrm{X}_{4}-$ факторы (предикторы) модели;

$\varepsilon-$ случайная ошибка модели.

Результаты расчетов приведены в таблице 4.

В первой модели в качестве независимых переменных $\mathrm{X}_{1}-\mathrm{X}_{4}$ использовались соответственно логарифмы целевой суммы, длительности сбора средств, количества спонсоров и размера среднего взноса. Во второй модели в качестве переменных $\mathrm{X}_{1}-\mathrm{X}_{3}$ использовались наличие заглавного видео и логарифмы количества новостей и количества комментариев, а в качестве $\mathrm{X}_{4}$ попеременно подставлялись три переменные, связанные с опытом, а для коммерческих проектов еще переменная соответствия вознаграждения. Для всех моделей приведены коэффициенты детерминации.

Уровень значимости всех регрессионных моделей был подтвержден на основании F-критерия Фишера. Оценка ошибок проводилась с помощью тестов Уайта и Голдфелда - Куандта, которые продемонстрировали наличие гетероскедастичностиㄹ․

\footnotetext{
${ }^{1}$ Переменные, связанные с предшествующим опытом автора: опыт вообще (9), негативный (10) и положительный (11), являются взаимозависимыми, поэтому для расчета значения фактора VIF, а также в регрессионных моделях они использовались по отдельности.

${ }^{2}$ Гетероскедастичность - неоднородность наблюдений статистической выборки, которая выражается в непостоянной дисперсии случайной ошибки модели $\varepsilon$ и приводит к неэффективности оценок случайных ошибок, полученных с помощью метода наименьших квадратов.
} 
В условиях гетероскедастичности коэффициенты $\beta$ остаются состоятельными и несмещенными, а значит, могут быть использованы для интерпретации, но вместо стандартных ошибок используются робастные стандартные ошибки, устойчивые к гетероскедастичности.

Таблица 4. Параметры регрессионных моделей

\begin{tabular}{|l|c|c|c|c|}
\hline \multirow{2}{*}{} & \multicolumn{2}{|c|}{ Коммерческие проекты } & \multicolumn{2}{c|}{ Общественные проекты } \\
\cline { 2 - 5 } & Коэф-т & SE & Коэф-т & SE \\
\hline Модель 1 & & & & \\
\hline Ln (Целевая сумма) & $-0,020^{\star}$ & 0,001 & $-0,072^{\star}$ & 0,003 \\
\hline Ln (Длительность) & 0,003 & 0,002 & $-0,024^{\star}$ & 0,005 \\
\hline Ln (Количество спонсоров) & $0,064^{\star}$ & 0,003 & $0,127^{\star}$ & 0,004 \\
\hline Ln (Средний взнос) & $0,094^{\star}$ & 0,002 & $0,032^{\star}$ & 0,003 \\
\hline Коэффициент детерминации $\left(\mathrm{R}^{2} \mathrm{adj}\right)$ & 0,965 & 0,077 & 0,658 & 0,274 \\
\hline Модель 2 & & & & \\
\hline Наличие заглавного видео & $0,027^{\star}$ & 0,008 & $-0,018$ & 0,011 \\
\hline Ln (Количество новостей) & $0,200^{\star}$ & 0,007 & $0,133^{\star}$ & 0,006 \\
\hline Ln (Количество комментариев) & $0,100^{\star}$ & 0,005 & $0,119^{\star}$ & 0,007 \\
\hline Наличие опыта & $0,160^{\star}$ & 0,016 & $0,165^{\star}$ & 0,013 \\
\hline Коэффициент детерминации $\left(\mathrm{R}^{2} \mathrm{adj}\right)$ & 0,447 & 0,312 & 0,232 & 0,413 \\
\hline Наличие негативного опыта & 0,039 & 0,020 & $0,062^{\star}$ & 0,017 \\
\hline Коэффициент детерминации $\left(\mathrm{R}^{2} \mathrm{adj}\right)$ & 0,435 & 0,315 & 0,213 & 0,418 \\
\hline Наличие успешного опыта & $0,261^{\star}$ & 0,021 & $0,230^{\star}$ & 0,017 \\
\hline Коэффициент детерминации $\left(\mathrm{R}^{2} \mathrm{adj}\right)$ & 0,454 & 0,310 & 0,247 & 0,409 \\
\hline Соответствие вознаграждения & $0,123^{\star}$ & 0,008 & & \\
\hline Коэффициент детерминации $\left(\mathrm{R}^{2} \mathrm{adj}\right)$ & 0,440 & 0.314 & & \\
\hline
\end{tabular}

Примечание: * $\mathrm{p}<0,001$. Для коэффициентов регрессии приведены робастные стандартные ошибки.

Гипотеза Н1 предполагает наличие связи между параметрами проекта и вероятностью успешного сбора средств.

Действительно, мы обнаружили обратную зависимость между размером целевой суммы и успешностью сбора средств для коммерческих $(\beta=-0,020)$ и общественных инициатив $(\beta=-0,072)^{3}$. Однако для коммерческих проектов это влияние значительно слабее, что указывает на то, что они имеют лучшую перспективу сбора более высоких сумм. Также обнаруживается прямая зависимость между количеством спонсоров, поддержавших проект, и размером среднего взноса и успешностью проекта. При

\footnotetext{
3 Здесь и далее все обсуждаемые статистики имеют значимость $\mathrm{p}<0,001$
} 
этом количество спонсоров для общественных проектов более значимо, чем для коммерческих $(\beta=0,127$ и $\beta=0,064$ соответственно), тогда как в отношении размера среднего взноса ситуация противоположная ( $\beta=0,032$ и $\beta=0,094)$. Это говорит о том, что общественные инициативы достигают успеха преимущественно за счет большого числа сравнительно малых взносов, тогда как в коммерческих проектах создаваемый продукт приобретается более узкой целевой аудиторией, но по стоимости, приближенной к рыночной, а потому более высокой. Длительность сбора средств не оказывает статистически значимого влияния на успешность коммерческих проектов, но отрицательно отражается на успешности общественных $(\beta=-0,024)$. Обнаруженная отрицательная зависимость согласуется с результатами исследований на зарубежных площадках [Mollic, 2014; Strickler, 2011] и может указывать на рассеивание внимания аудитории и эффект размывания ответственности, когда потенциальный спонсор, видя большой срок проекта, рассчитывает, что его поддержит кто-нибудь другой [Kuppuswamy, Bayus, 2013; Burtch at el., 2013]. В то же время отсутствие влияния длительности на успешность коммерческих инициатив говорит о том, что в этой группе гораздо более значимым оказывается качество самой идеи: невостребованные инициативы не достигнут успеха при любых сроках проекта, а у хорошо проработанных проектов отсутствует эффект размывания ответственности, потому что спонсоры, заинтересованные в создаваемом продукте, не откладывают его покупку. Примечательно, что, в отличие от американской краудфандинговой площадки Kickstarter, изученной коллегами [Strickler, 2011], аудитория Planeta.ru толерантно относится к сравнительно длинным срокам сбора в 2-2,5 месяца, которые являются наиболее распространенными.

Таким образом, пункты а), б) и в) гипотезы Н1 полностью подтверждены, тогда как пункт г) полностью опровергнут. В целом гипотезу Н1 можно считать подтвержденной, поскольку установлена связь рассмотренных параметров и успешности проекта.

Гипотеза $\mathbf{H 2}$ предполагает наличие связи между качеством информационного сопровождения проекта и вероятностью успешного сбора средств.

Действительно, мы обнаружили значительное положительное влияние количества новостей и комментариев по проекту 
на успешность и коммерческих $(\beta=0,200$ и $\beta=0,100$ соответственно) и общественных инициатив $(\beta=0,133$ и $\beta=0,119)$, что подчеркивает важность выстраивания регулярной коммуникации с аудиторией. При этом новости, которые, в отличие от комментариев, всегда инициируются автором, имеют заметно большее значение. Вместе с тем наличие заглавного видео положительно воздействует на успешность только коммерческих проектов $(\beta=0,027)$, тогда как для общественных инициатив статистически значимого влияния не обнаружено. В целом можно заключить, что для коммерческих проектов качественное информационное сопровождение со стороны автора гораздо более важно, чем для общественных. Итак, пункты а) и б) гипотезы Н2 подтверждены полностью, а пункт в) только частично.

Гипотезой Н3 предусмотрено наличие влияния предшествующего опыта автора в организации краудфандинговых инициатив на успешность последующих проектов. Обнаружено, что наличие опыта в краудфандинге вообе, а в особенности - наличие успешного опыта, оказывает заметное положительное влияние на успех как коммерческих ( $\beta=0,160$ и $\beta=0,261$ соответственно), так и общественных инициатив $(\beta=0,165$ и $\beta=0,230)$. При этом наличие негативного опыта не оказывает отрицательного влияния. Более того, для социальных проектов получен статистически значимый положительный коэффициент $(\beta=0,062)$, что, на наш взгляд, говорит о положительном влиянии любого опыта как такового. Таким образом, гипотеза Н3 подтверждена: в организации краудфандинговых проектов явно наблюдается эффект обучения, а наличие неудачного опыта не уменьшает шансы на будущий успех.

Гипотеза Н4 предполагает значимость для успеха коммерческих проектов соответствия вознаграждения вкладу спонсора. Данные регрессионного анализа явно подтверждают наличие такой положительной связи $(\beta=0,123)$. Это указывает на то, что мотивы финансирования коммерческих инициатив через краудфандинг отличаются от таковых для благотворительных и общественных проектов, а значит, апеллирование к аудитории за инвестициями в таких случаях должно быть подкреплено соответствующими вознаграждениями. Этот вывод кажется самоочевидным, однако, как выяснилось, далеко не для всех. В ходе исследования мы обнаружили более 500 коммерческих 
инициатив, авторы которых не предложили спонсорам вознаграждений, соизмеримых с размером вклада. Практически все они (98\%) оказались безуспешными.

Вместе с тем среди этих инициатив было несколько, на наш взгляд, достаточно проработанных и качественных проектов, которые не могли предложить свой продукт спонсорам по объективным причинам. Например, из-за несовпадения аудитории потребителей будущей продукции с аудиторией краудфандинговой площадки или высокой стоимости продукции, значительно превышающей размер взносов и др. Для таких инициатив вместо нефинансовой модели краудфандинга, применяемой на Planeta.ru, на наш взгляд, больше подошла бы долговая или долевая модель, первая подразумевает вознаграждение спонсоров через выплату процентов на сумму вложений, вторая - предоставление доли в капитале создаваемой компании. Тогда была бы соблюдена материальная заинтересованность спонсоров соответственно размеру их взноса и уровню риска. Гипотезу $\mathbf{H 4}$ можно считать полностью подтвержденной.

\section{Заключение}

Проведенный анализ указывает на перспективность использования краудфандинга для финансирования коммерческих и общественных инициатив. С помощью корреляционно-регрессионного анализа были подтверждены большинство положений тестируемых гипотез о влиянии на успешность проекта его основных параметров, задаваемых при запуске, а также информационного сопровождения на этапе его реализации.

Показано, что качественные коммерческие инициативы имеют лучшие перспективы сбора больших сумм, а их успешность в большей степени зависит от усилий автора по качественному информационному сопровождению. Вместе с тем доказано, что реализация коммерческих краудфандинговых инициатив должна обеспечивать обоюдовыгодный экономический обмен, и поэтому должно соблюдаться соответствие вознаграждений размеру взносов спонсоров.

В работе прослеживается эффект обучения авторов по мере приобретения опыта в краудфандинге. А также показано, что недостаточная информированность участников краудфандинговых отношений о нюансах этого процесса порой приводит к тому, что качественные высокотехнологичные инициативы 
могут не найти отклика у аудитории из-за неверно выбранного краудфандингового механизма. Здесь отметим, что отчасти этот выбор осложняется из-за законодательной неурегулированности долевой модели краудфандинга, при которой спонсорам в качестве вознаграждения предлагается доля в капитале создаваемой компании. Преодоление этих ограничений будет способствовать более эффективному использованию краудфандинга для финансирования молодых инновационных компаний.

\section{Литература/ References}

Амосов Ю. Четыре мобильности: вызовы России в 2015-2030 гг. Доклад. Аналитический центр при Правительстве России. 2014. 76 с.

Amosov, Yu. (2014). Four mobility: Challenges for Russia in 2015-2030. Analytical report. Analytical Center for the Government of the Russian Federation. 76 p. (In Russ.).

Салтыков Б.Г., Гордеев М.Н. Краудфандинг как инструмент венчурного инвестирования в России и за рубежом // Инновации. 2016. № 4 (210). С. 51-57.

Saltykov, B. G., Gordeev, M.N. (2016). Crowdfunding as a tool of venture investment in Russia and abroad. Innovatsii. Innovations. No. 4 (210). Pp. 51-57. (In Russ.).

Соловьев A. Стартап барометр 2018. Исследование рынка технологического предпринимательства в России. 2018. 22 с.

Solov'ev, A. (2018). Startup barometer 2018. Overview of the Russian startup market. 22 p. (In Russ.).

Burtch, G., Ghose, A., and Wattal, S. (2013). An Empirical Examination of the Antecedents and Consequences of Contribution Patterns in Crowd-Funded Markets. Information Systems Research. Vol. 24. No. 3. Pp. 499-519.

Chan, C.S.R. (2017). Crowdfunding Innovative Ideas: How incremental and Radical Innovativeness Influence Funding Outcomes. Entrepreneurship Theory and practice

Cordova, A., Dolci, J., Gianfrante, G. (2015). The determinants of crowdfunding success: evidence from technology projects. Procedia - Social and Behavioral Sciences. Vol. 181. Pp. 115-124.

Field, A. (2005). Discovering Statistics with SPSS. London: SAGE.

$H s u, D$. (2007) Experienced entrepreneurial founders, organizational capital, and venture capital funding. Research Policy. Vol.36. Pp. 722-741.

Kuppuswamy, V., Bayus, B.L. (2013) Crowdfunding creative ideas: the dynamics of project backers in kickstarter. UNC Kenan-Flagler Research Paper. No 15. 42 p.

Lynn, J. (2013) Democratise investment to help the many not the few. The Telegraph

Mollick, E. (2014). The Dynamics of Crowdfunding: Determinants of Success and Failure. Journal of business Venturing. Vol. 29. No.1. Pp. 1-16.

Mollick, E., Robb, A. (2016a). Democratizing Innovation and Capital Access: The Role of Crowdfunding. California Management Review. Vol. 58. No. 2. Pp. 72-87. 
Mollick, E., Ethan, R., (2016b). Containing Multitudes: The Many Impacts of Kickstarter Funding. Available at: https://ssrn.com/abstract=2808000 (accessed 21.05.2018).

Schwienbacher, A., Larralde, B. (2012). Alternative Types of Entrepreneurial Finance. In Cumming, D.J. (ed.). The Oxford handbook of entrepreneurial finance. Oxford: Oxford University Press. 2012. 23 p.

Shane, S., Cable, D. (2002). Network Ties, Reputation, and the Financing of New Ventures. Management Science. Vol. 48. No 3. Pp. 364-381.

Strickler, Y. (2011). Shortening the Maximum Project Length. Kickstarter PBC. Available at: https://www.kickstarter.com/blog/shortening-the-maximum-projectlength (accessed 21.05.2018).

Статья поступила 24.05.2019.

Для цитирования: Гордеев М.Н. Предикторы успешности краудфандингового проекта // ЭКО. № 8. С. 179-192. DOI: 10.30680/ECO0131-7652-20198-179-192.

For citation: Gordeev, M.N. (2019). Predictors of Success in a Crowdfunding Project. ECO. No. 8. Pp. 179-192. (In Russ.). DOI: 10.30680/ECO0131-7652-20198-179-192.

\section{Summary}

Gordeev, M.N., National Research University Higher School of Economics, Moscow

\section{Predictors of Success in a Crowdfunding Project}

Abstract. The article discovers determinants of a successful fundraising via crowdfunding. The undertaken analysis is based on the method of correlation and regression. Drawing on a dataset of over 12,000 projects carried out in Russia, this paper reveals the relation between the success of the project and the initial launch parameters set by the author, as well as the information support during the project. The study identifies the learning effect which appears in raising chances for a successful fundraising for authors with previous crowdfunding experience. Also a correlation between comparability of the sponsor's reward to his pledge and the success of commercial initiatives has been established. The research gives an indepth description of the crowdfunding and the underlying processes. Special attention has been paid to the crowdfunding usage as an instrument for venture fundraising.

Keywords: Crowdfunding; public funding; successful fundraising; Planeta.ru 